\title{
OS IDOSOS E A COMPLEXIDADE DOS REGIMES TERAPÊUTICOS
}

Com o rápido processo de transição demográfica e do envelhecimento populacional em nosso país, o aumento da prevalência das doenças crônico-degenerativas torna de extrema importância questões como polifarmácia, iatrogenia, interações medicamentosas e ocorrência de eventos adversos (EAs) a que estão expostos os idosos. A frequência do uso de medicamentos nesta faixa etária é elevada, com valores entre $60 \%$ a $90 \%$, dos quais um terço utiliza cinco ou mais medicações simultaneamente. Por um lado, há consumo excessivo de produtos de automedicação, medicamentos supérfluos ou mal indicados, e por outro lado, a subutilização ou o uso inadequado de produtos eficazes e essenciais para o controle das doenças. Entre os diversos fatores preditivos da polifarmácia em idosos brasileiros já estudados estão a idade avançada, o sexo feminino, os piores índices de autoavaliação da saúde e a depressão ${ }^{1-4}$.

Os idosos apresentam, com frequência, múltiplas comorbidades e redução da reserva funcional de órgãos e sistemas. A farmacoterapia nesta população tem suas peculiaridades, por redução da massa muscular e da água corporal, alterações do metabolismo hepático, dos mecanismos homeostáticos, bem como da capacidade de filtração e de excreção renal. Disso decorre a dificuldade de eliminação de metabólitos, o acúmulo de substâncias tóxicas ao organismo e a vulnerabilidade à ocorrência de reações adversas. As alterações neurossensoriais (déficit visual, hipoacusia) e o declínio cognitivo tornam ainda mais complexos e difíceis a adesão e o uso apropriado dos produtos farmacêuticos nesta população. O uso da linguagem técnica gera dificuldade na compreensão. Embora a exposição a múltiplos fármacos (polifarmácia) não seja necessariamente sinônimo de prescrição inapropriada ou iatrogenia, aumentam os riscos acima descritos. Além disso, metade dos indivíduos em uso de seis ou mais diferentes fármacos, usualmente recebem as prescrições de três ou mais diferentes médicos, o que aumenta a possibilidade de interações medicamentosas e de desarticulação entre as condutas, agravando a complexidade das prescrições.

Oportuno, original, de temática atual e muito relevante 0 artigo de Acurcio FA et al., publicado nesta edição da RAMB, sobre Índice de Complexidade Terapêutica (ICT) de prescrições médicas a uma amostra populacional de idosos. Foi realizado um estudo de corte transversal por inquérito domiciliar sobre o perfil de utilização de medicamentos por aposentados brasileiros, em amostra aleatória simples de 667 indivíduos acima de 60 anos de idade, já aposentados e vinculados ao regime de Previdência Social/INSS/MPAS, moradores de Belo Horizonte, MG, em 2003. Os idosos foram entrevistados por farmacêuticos, utilizando questionário padronizado. A prevalência do uso de medicamentos foi de $90,1 \%$, sendo significativamente maior entre as mulheres $(93,4 \%)$ do que entre os homens $(84,3 \%)$ que utilizavam em média 4,6 $\pm 3,2$ e 3,3 $\pm 2,6$ produtos, respectivamente $(p<0,001)$. Os princípios ativos mais usados pelos idosos pertenciam ao sistema cardiovascular, sistema nervoso, trato alimentar e do metabolismo. ${ }^{1}$ Em análise posterior, foram investigados os
ICTs e os fatores sociodemográficos e dados de saúde associados ao mesmo. Entre 438 (65,7\%) dos entrevistados em uso de medicação prescrita nos 15 dias anteriores à pesquisa, 377 preencheram critérios de inclusão para a análise de complexidade terapêutica. Quase 60\% dos idosos relataram quatro ou mais doenças, sendo mais prevalentes os problemas de visão $(82,8 \%)$, hipertensão arterial (70,6\%) e depressão (36,3\%). Nos 15 dias anteriores à entrevista, a média de consumo foi igual a cinco produtos farmacêuticos por pessoa, sendo a metade em uso sem prescrição médica, 22,3\% dos participantes incluídos na análise relataram o não uso de algum dos medicamentos prescritos (má adesão). O ICT foi calculado apenas para os medicamentos prescritos, por uma equação mais abrangente que contempla o número de fármacos, a posologia diária, as ações mecânicas necessárias ao uso e as instruções contidas nas prescrições. 0 ICT variou de 1 a 24, tendo média igual a 6,1 e mediana 5,0. Foi observada associação significativa entre o ICT com os seguintes fatores: maior número de medicamentos em uso, maior número de doenças referidas, maior número de consultas médicas em um ano, pior autopercepção da saúde, menor escolaridade, menor benefício recebido do INSS. A não adesão também se mostrou associada à maior complexidade do regime terapêutico indicado.

O ICT estima o grau de dificuldade que os idosos enfrentam para cumprir e seguir suas prescrições e o alto grau de complexidade terapêutica pode resultar em perda da autonomia e independência. Os autores sugerem a possibilidade de que aqueles com pior condição econômica e menor escolaridade tenham piores condições de saúde, resultando na prescrição de esquemas terapêuticos mais complexos e constituindo grupo mais vulnerável às complicações advindas. A despeito das possíveis explicações para as associações encontradas, sugere-se que os médicos que os atendem, em especial aqueles que devem, em primeiro momento, "gerenciar" seus cuidados de saúde, sejam eles geriatras, clínicos, cardiologistas que busquem a adequação do regime terapêutico com o objetivo de facilitar o autocuidado, estimulando sempre que possível a independência.

Os dados encontrados neste estudo reforçam a necessidade de que a assistência farmacêutica ao idoso seja uma preocupação e constitua prioridade às políticas de saúde, com o objetivo de garantir a esse segmento populacional o acesso aos medicamentos adequados e o seu uso com qualidade. Os autores sugerem que, embora se deva ter cautela nas generalizações, é razoável supor que o panorama apresentado possa ser semelhante em outros locais no Brasil. A preocupação com o uso inapropriado de medicamentos e problemas advindos deve estimular a criação de listas de substâncias a serem contraindicadas, evitadas ou usadas apenas em circunstâncias excepcionais em idosos em nosso país, como já acontece em alguns países europeus, no Canadá e nos Estados Unidos. Nestas listas há recomendações especiais relacionadas a fármacos como os benzodiazepínicos, os hipoglicemiantes orais de meia-vida longa, os barbitúricos, os antidepressivos com forte ação anticolinérgica, 
alguns analgésicos opioides, as associações de antidepressivos a antipsicóticos, a indometacina, agentes anti-inflamatórios e relaxantes musculares, dentre outros ${ }^{2}$.

No campo da investigação, faltam melhores critérios para a inclusão dos "verdadeiros idosos" de nossa prática clínica nos diversos ensaios clínicos, diretrizes, consensos terapêuticos das diversas especialidades. E uma boa sugestão, como estratégia para aprimorarmos as atitudes frente à fragilidade e vulnerabilidade destes nossos pacientes, é incluirmos os farmacêuticos nos serviços de atendimento, centros especializados e instituições de longa permanência para idosos, fazendo parte das equipes interdisciplinares geriátricas. Como referência à sugestão, um ensaio clínico recente ${ }^{5}$ avaliou a presença e intervenção do profissional farmacêutico em equipe de atendimentos hospitalares a idosos muito idosos (acima de 80 anos), e encontrou importante e significativa redução de visitas a unidades de pronto atendimento (47\%), e de readmissão por problemas relacionados a medicamentos (80\%) no período de um ano de seguimento.

- Há medidas importantes a serem seguidas para o aprimoramento da qualidade da prescrição médica do paciente idoso:

- Estímulo ao emprego, sempre que possível, de medidas não-farmacológicas;

- Acompanhamento, com revisão periódica, do conjunto dos medicamentos e de seus possíveis EAs e interações;

- Preferência por monoterapias, em vez de associações em doses fixas;

- Prescrição dos fármacos de eficácia comprovada por evidências científicas neste grupo etário:

- Suspensão do uso de produtos fúteis e sem eficácia comprovada, inclusive dos complexos vitamínicos inapropriados;

- Verificação da compreensão da prescrição e das orientações (devem ser sempre escritas e legíveis);

- Simplificação dos esquemas de administração;

- Atenção aos custos.
Além destas questões, é muito importante ressaltar a necessidade de interação e da boa comunicação entre os médicos das diversas especialidades e de todos os profissionais de saúde que os atendem.

Maira Tonidandel Barbosa Coordenadora da Pós-graduação em Geriatria - Hospital Mater Dei e Faculdade de Ciências Médicas de Minas Gerais, Belo Horizonte, MG

\section{Correspondência}

Rua Alvarenga Peixoto, n 711 - Apto. 703 - Bairro de Lourdes CEP: 30180-120

Belo Horizonte - MG

email: mairatonidandel@terra.com.br

\section{Referências}

1. Ribeiro $\mathrm{AQ}$, Rozenfeld $\mathrm{S}$, Klein $\mathrm{CH}$, César $\mathrm{CC}$, Acurcio FA. Inquérito sobre uso de medicamentos por idosos aposentados, Belo Horizonte, MG. Rev Saúde Pública. 2008;42(4):724-32.

2. Gorzoni ML, Fabbri RMA, Pires SL. Critérios de Beers-Fick e medicamentos genéricos no Brasil. Rev Assoc Med Bras. 2008;54(4):353-6.

3. Rozenfeld S. Prevalência, fatores associados e mau uso de medicamentos entre os idosos: uma revisão. Cad Saúde Pública. 2003;19(3):717-24.

4. Loyola Filho AI, Uchoa E, Firmo JOA, Lima-Costa MF. Estudo epidemiológico de base populacional sobre uso de medicamentos entre idosos na Região Metropolitana de Belo Horizonte, Minas Gerais, Brasil. Cad Saúde Pública. 2006;22(12):2657-67.

5. Gillespie U, Alassaad A, Henrohn D, Garmo H, Hammarlund-Udenaes M, Toss $\mathrm{H}$, et al. A Comprehensive pharmacist intervention to reduce morbidity in patients 80 years or older - a randomized controlled trial. Arch Intern Med. 2009;169(9):894-900.

6. Acurcio FA, Silva AL, Ribeiro AQ, Rocha NP, Silveira MR et al. Complexidade do regime terapêutico prescrito para idosos, Belo Horizonte, MG. Rev. Assoc. Med. Bras. 2009; 55(4) 468-74. 